\title{
Differential Salmonella survival against communities of intestinal amoebae
}

\author{
Hans Wildschutte and Jeffrey G. Lawrence \\ Department of Biological Sciences, University of Pittsburgh, Pittsburgh, PA 15235, USA
}

\begin{abstract}
Correspondence
Jeffrey G. Lawrence

jlawrenc@pitt.edu
\end{abstract}

Received 20 October 2006

Revised 26 January 2007

Accepted 2 February 2007
Predation from intestinal amoebae may provide selective pressure for the maintenance of high genetic diversity at the Salmonella enterica rfb locus, whereby serovars better escape predators in particular environments depending on the O-antigens they express. Here, the hypothesis that amoebae from a particular intestinal environment collectively prefer one serovar over another is tested. Collections of Acanthamoeba, Tetramitus, Naegleria and Hartmannella were isolated from the intestinal tracts of several vertebrate hosts, including bullfrog tadpoles, goldfish, turtles and bearded dragons, and their feeding preferences were determined. Congeneric amoebae from the same environment had significantly similar feeding preferences. Strikingly, even unrelated amoebae - such as Naegleria and Tetramitus from goldfish - also had significantly similar feeding preferences. Yet amoebae isolated from different environments showed no similarity in prey choice. Thus, feeding preferences of amoebae appear to reflect their environment, not their taxonomic relationships. A mechanism mediating this phenotypic convergence is discussed.

\section{INTRODUCTION}

Given their enormous numbers in nearly every ecosystem, the control of bacterial population sizes and growth rates ranks as one of the most powerful regulators of biomass on the planet. The numbers of viable bacteria - residing in the water column, soil and subsurface sediments - have been estimated to be $4-6 \times 10^{30}$ cells, with turnover times measured in days (Whitman et al., 1998); this constant replacement of bacteria by cell division suggests that bacterial mortality occurs at a high rate. Both amoebae and phage contribute to bacterial mortality in these environments and are effective predators in controlling bacterial populations, whose cell counts increase significantly in the absence of either predator (Jjemba, 2001; Peduzzi \& Schiemer, 2004; Pernthaler, 2005; Ronn et al., 2002). Phages are specialized predators which recognize their prey primarily through specific motifs on outer-membrane proteins or carbohydrates, ensuring that phage replication occurs in a compatible host, while amoebae are generalist scavengers which prey upon bacteria relatively indiscriminately.

Bacterial abundance in intestinal environments has been

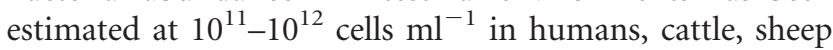

\footnotetext{
Abbreviations: DS, diversifying selection; FDS, frequency-dependent selection; FLA, free-living Acanthamoeba.

The GenBank/EMBL/DDBJ accession numbers for the amoebal DNA sequences determined in this paper are EF378666-EF378696; those for the bacterial sequences are EF491825-EF491854.

A supplementary figure is available with the online version of this paper.
}

and pigs (Whitman et al., 1998). Dominant species of bacteria include members of the Gram-positive taxa Bacteroides, Clostridium and Lactobacillus, whereas minor constituents are Gram-negative enteric bacteria such as Escherichia coli, Salmonella enterica and Citrobacter freundii (Eckburg et al., 2005; Ley et al., 2006; Palmer et al., 2006). As minor constituents of the intestinal flora, changes in the Gram-negative members of this community would be unlikely to affect the behaviour of the predators, which feed primarily on Gram-positive bacteria. As such, we can more directly assess how changes in predator behaviour may alter the distribution and abundance of Gram-negative prey, decoupling changes in predator populations from changes in their prey populations.

A characteristic structure of a Gram-negative bacterium is the membrane-attached lipopolysaccharide (LPS) component of its cell wall. Being the most abundant molecule on the surface of the bacterial cell, LPS is a likely structure used by predators to recognize their prey (Matz \& Kjelleberg, 2005; Wildschutte et al., 2004). The O-antigen is the outermost leaflet of LPS; it is anchored to the outer-membrane-bound lipid A via the highly conserved core oligosaccharide (Heinrichs et al., 1998; Holst \& Brade, 1992). Strains of Salmonella enterica exhibit more than 70 different $\mathrm{O}$-antigens that help define serological groups (Popoff, 2001). The closely related enteric bacteria Escherichia coli and Citrobacter freundii also show great diversity in O-antigen types, and display at least 170 (Whitfield \& Roberts, 1999) and 45 (Knirel et al., 2002) different O-antigens, respectively. The enzymes responsible for O-antigen synthesis in Salmonella and other enteric bacteria are encoded by the $r f b$ operon, 
which exhibits extensive genetic diversity (Liu et al., 1991; Milkman et al., 2003; Stenutz et al., 2006; Xiang et al., 1993). High genetic diversity at the $r f b$ locus is maintained because no one allele, or $\mathrm{O}$-antigen, confers the highest fitness among serovars; therefore, no allele appears to have initiated a selective sweep, as has occurred at other loci (Guttman \& Dykhuizen, 1994; Hermisson \& Pennings, 2005).

Previously, we hypothesized that predation from intestinal amoebae provides selective pressure for maintaining $r f b$ genetic diversity among Salmonella (Wildschutte et al., 2004); that is, a serovar may better escape predators in a particular environment by virtue of the $\mathrm{O}$-antigen it possesses, and other serovars flourish in environments harbouring different predators. Supporting this diversifying selection (DS) model, we showed that intestinal amoebae consumed Salmonella serovars at different rates if they expressed dissimilar Oantigens (Wildschutte et al., 2004). Furthermore, predators could discriminate among Salmonella that differed solely at their $\mathrm{O}$-antigen, so that the $\mathrm{O}$-antigen itself is sufficient to elicit a feeding preference among predators (Wildschutte et al., 2004). Conventional models for the maintenance of antigenic diversity invoke frequency-dependent selection (FDS) (Reeves, 1995; Wang et al., 1992); here, rare serotypes persist because they allow for infection of naïve hosts. But FDS is entirely incompatible with the well-established phenomenon of serovar-host specificity - the clinical observation whereby serovars expressing a certain $\mathrm{O}$-antigen usually infect and cause disease in particular hosts - whereas our DS model cleanly explains this otherwise puzzling aspect of bacterial natural history.

A prediction of the DS model is that predators in a particular environment will collectively prefer one serovar over another based on the identity of the prey's $\mathrm{O}$-antigen. This may occur if (a) co-resident amoebae are related and simply share ancestral feeding preferences, or (b) unrelated predators share feeding preferences because the environment influences prey choice. Alternatively, if amoebae in a particular environment do not share feeding profiles, then no single serovar would have an advantage in escaping all co-resident predators and $r f b$ genetic diversity could not be maintained by this DS model. To discriminate among these alternatives, we isolated multiple amoebae from different environments, identified them based on the sequences of their 18S rDNA loci, and tested their feeding preferences to determine if bacterial serovars could escape communities of predators.

\section{METHODS}

Media and growth conditions. The bacterial isolates described below, or strains from the SARB collection (Boyd et al., 1993) SARB1 (serotype Agona O : 1,4,12), SARB2 (Anatum O :3,10), SARB3 (Brandenberg $\mathrm{O}: 1,4,12$ ), SARB8 (Decatur O:6,7), SARB20 (Emek $\mathrm{O}: 8,20$ ), SARB30 (Montevideo O:6,7), SARB36 (Newport O:6,8), SARB52 (Pullorum O:1,9,12), SARB59 (Senftenberg O:1,3,19) were grown on solid LB medium overnight at $37^{\circ} \mathrm{C}$. For propagation of amoebae, NM medium was prepared as $15.5 \mathrm{mM}$ potassium phospate $\mathrm{pH}$ 7.5, $0.2 \%$ peptone, $0.2 \%$ glucose, $2.0 \%$ agar; amoebae and bacteria were incubated at $34{ }^{\circ} \mathrm{C}$ during predation experiments.
NM-LG (low glucose) medium was prepared as NM except with $0.02 \%$ glucose and $1.5 \%$ agar. SBG sulfa enrichment medium, XLT4 medium and MacConkey-lactose medium were purchased from Difco and prepared as directed. LB was prepared as $1 \%$ tryptone, $0.5 \%$ yeast extract, $0.5 \% \mathrm{NaCl}$ and $1.2 \%$ agar.

Isolation and identification of intestinal amoebae. Bullfrog tadpoles (Rana catesbeiana) were collected from Geneva Pond \#1 in Crawford county Pennsylvania; goldfish (Carassius auratus auratus) were purchased from a local pet store; turtles (Trachemys scripta) were purchased from Ward's Scientific Supply House. Lower intestinal contents were removed via sterile dissection into sterile water; no amoebae were collected from outside the intestinal lumen. Amoeba cysts were separated from bacteriophage by differential centrifugation. Aliquots of 10-100 $\mu \mathrm{l}$ of the intestinal sample were spread on NM medium seeded with $10^{8}$ Salmonella enterica serovar Typhimurium LT2 cells as food. Plates were incubated at $34{ }^{\circ} \mathrm{C}$ to allow for growth of amoebae within bacterial lawns. Protozoan cysts were collected from cleared plaques, diluted and reisolated to ensure purity. Bearded dragons (Pogona barbata) were pets of a colleague; isolation of their intestinal amoebae was performed as above except using freshly collected faecal samples as starting material. Chromosomal DNA was isolated from amoebae using the DNeasy kit from Qiagen. An internal fragment of the 18S rDNA gene was amplified using primers U509F (5'-ACTCGAGTGCCAGCAGCCGCGGTAA-3') and E1789R (5'TCCGCAGGTTCACCTACGGA-3'), and the nucleotide sequences of both strands of the resulting product were determined using ABI310 and ABI-3100 sequencers. Strains of amoebae obtained are listed in Table 1; DNA sequences have been deposited in GenBank and assigned accession nos EF378666-EF378696.

Isolation and identification of intestinal bacteria. Aliquots of $10-200 \mu \mathrm{l}$ of intestinal/faecal samples from turtles, bearded dragons and goldfish were placed in SBG sulfa enrichment medium and incubated overnight at $37^{\circ} \mathrm{C}$. Cultures were then diluted and plated on XLT4 or MacConkey-lactose media. Black or pink colonies, respectively, were picked and streaked for single colonies on LB. Bacteria from a screeching owl (Otus asio), a resident of the National Aviary in Pittsburgh, were also isolated from freshly collected faecal samples. An internal fragment of the bacterial $16 \mathrm{~S}$ rDNA gene was amplified using primers rp1 (5'-CCCGGGATCCAAGCTTACGGTTACCTTGTTACGACTT-3') and fd2 (5'-CCGAATTCGTCGACAACAGAGTTTGATCATGGCTCAG-3'). A $1.4 \mathrm{~kb}$ band was routinely produced and the sequences of both strands were determined using ABI-310 and ABI-3100 sequencers. Strains of bacteria obtained are listed in Table 1 and DNA sequences have been deposited in GenBank and assigned accession numbers EF491825-EF491854.

Line tests and fitness calculations. The procedure for line tests was modified from the protocol of Wildschutte et al. (2004). Eight equally spaced lines were streaked on NM or NM-LG medium from the centre of the plate outward; four replicate lines of two strains were struck on each plate. Plates were incubated at $37^{\circ} \mathrm{C}$ until lines were fully grown. A total of $10^{4}$ protozoan cysts (numbers were determined via direct counting on a haemocytometer) in $10 \mu$ of $0.9 \% \mathrm{NaCl}$ was added to the centre of the plate on a sterile paper disk and plates were incubated at $34{ }^{\circ} \mathrm{C}$ to allow amoebae to germinate and consume the bacteria. Plates were photographed every $6 \mathrm{~h}$ and predation rates were determined using the distance of feeding front relative to the line's starting position. Regressions were calculated for distance consumed vs time (typically, $\left.R^{2}>0.95\right)$. The significance of the difference between the sets of four slopes per strain was determined using a $t$-test.

To assay fitness differences, all 36 (9 strains) or 10 ( 5 strains) pairwise comparison plates were examined. Overall consumption rates were calculated as mean slopes for the four replicates on each plate, which were then averaged across the independent pairwise competition 
Table 1. Environmental isolates of amoebae and bacteria

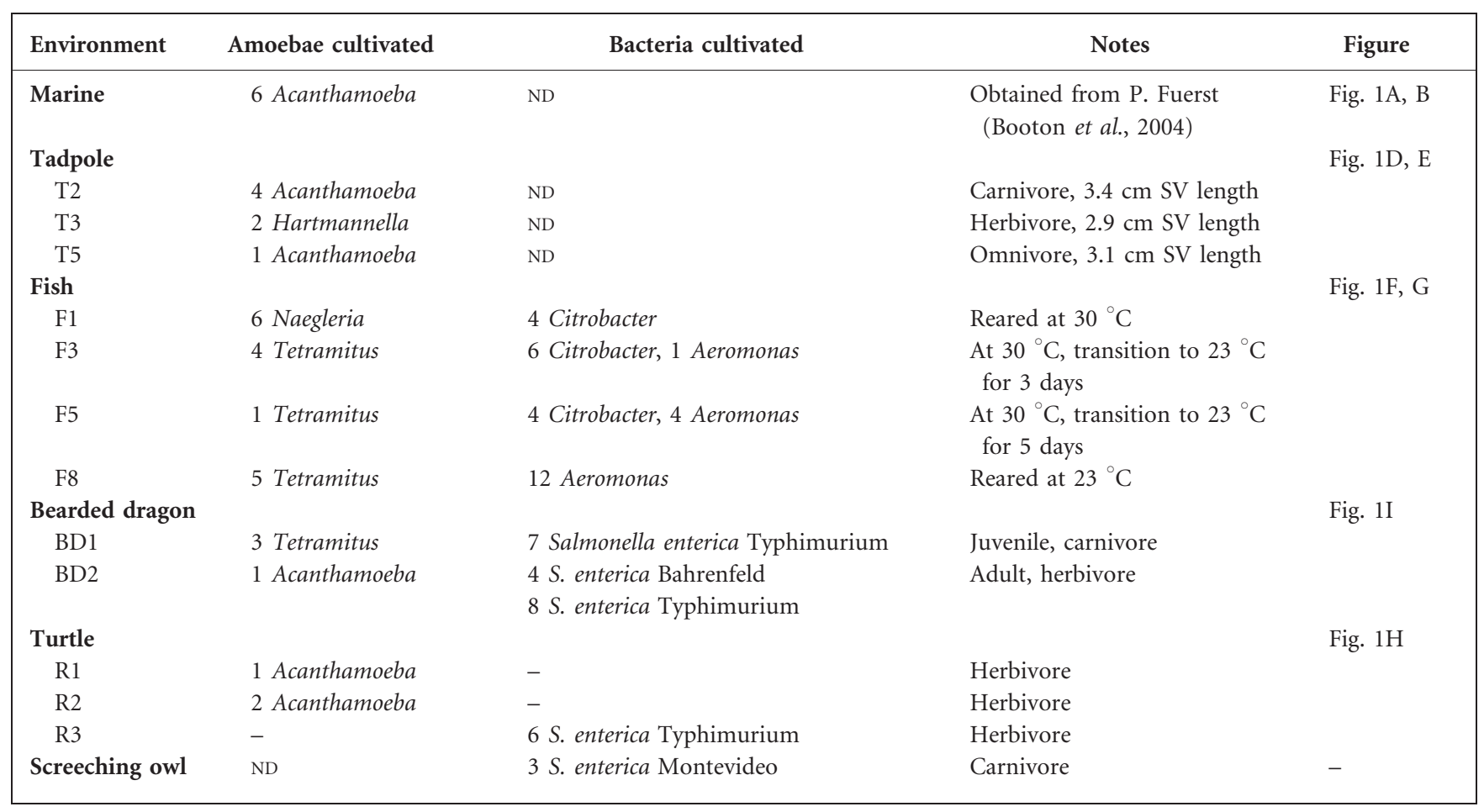

ND, Not determined.

plates bearing that strain. Cell density in bacterial lines was estimated as described by Wildschutte et al. (2004). Overall fitness values were calculated by multiplying the overall rate of consumption $\left(\mathrm{mm}^{2} \mathrm{~h}^{-1}\right)$ by the cell density (cells $\mathrm{mm}^{-2}$ ), normalizing corrected consumption rates $\left(\right.$ cells $\mathrm{h}^{-1}$ ) to the value of the least-preferred strain to obtain fitness. In addition, the robustness of the fitness hierarchy was validated by consistency of the overall relationships with the results of individual pairwise competition plates; that is, an overall hierarchy of $\mathrm{A}>\mathrm{B}>\mathrm{C}$ was validated by individual competition plates having yielded $\mathrm{A}>\mathrm{B}, \mathrm{B}>\mathrm{C}$ and $\mathrm{A}>\mathrm{C}$.

Feeding preference comparisons. The Pearson correlation coefficient $(R)$ was determined for each pairwise comparison of feeding preferences. For a collection of more than two sets of feeding preferences, an average value for $R\left(R_{\text {Average }}\right)$ was determined as simple arithmetic mean of the individual pairwise $R$ values. To determine if $R_{\text {Average }}$ were significantly different from zero, rates of predation were randomly assigned to prey and $\bar{R}_{\text {Average }}$ was computed for these randomized data. The significance of $R_{\text {Average }}$ for the observed data was computed as the number of randomized sets of comparisons with a value of $\bar{R}_{\text {Average }}$ that met or exceeded it. $P$-values were determined from 10000000 randomization trials.

\section{RESULTS}

\section{Related amoebae have similar feeding preferences}

Feeding preferences of amoebae represent their 'search image' for prey; by definition, amoebae eat bacteria matching this search image more quickly than bacteria that do not. These preferences may be a variable trait among amoebae populations if different members of the same species search for different prey. If feeding preferences change in this way, then otherwise genotypically and ecologically similar amoebae inhabiting the same intestinal environment may not recognize the same prey. As a result, no single O-antigen would allow one Salmonella serovar to escape all predators more efficiently than other serovars. Alternatively, generalist amoeba predators could retain the same feeding preferences if altering them provided no benefit to the amoeba, regardless of how these preferences affect their prey populations.

To discriminate between these alternatives, we obtained six strains of free-living Acanthamoeba (FLA), kindly provided by Paul Fuerst (Department of Molecular Genetics, Ohio State University, Columbus, Ohio, USA). These amoebae were isolated from a marine environment and were $>99 \%$ identical at their $18 \mathrm{~S}$ rDNA loci (Booton et al., 2004). The feeding preferences of the FLA-1 amoebae were determined using nine serotypically diverse strains of Salmonella from the SARB collection. The predator's ability to consume prey was measured using line tests as described above in Methods and as employed previously (Wildschutte et al., 2004). Among the nine strains tested, strain SARB52 (serovar Pullorum expressing the 1,9,12 Oantigen) was consumed the most slowly and was assigned a fitness value of 1.0 (Fig. 1A). The remaining strains were reproducibly consumed more quickly, indicating that the FLA-1 amoeba can discriminate among Salmonella serovars. 

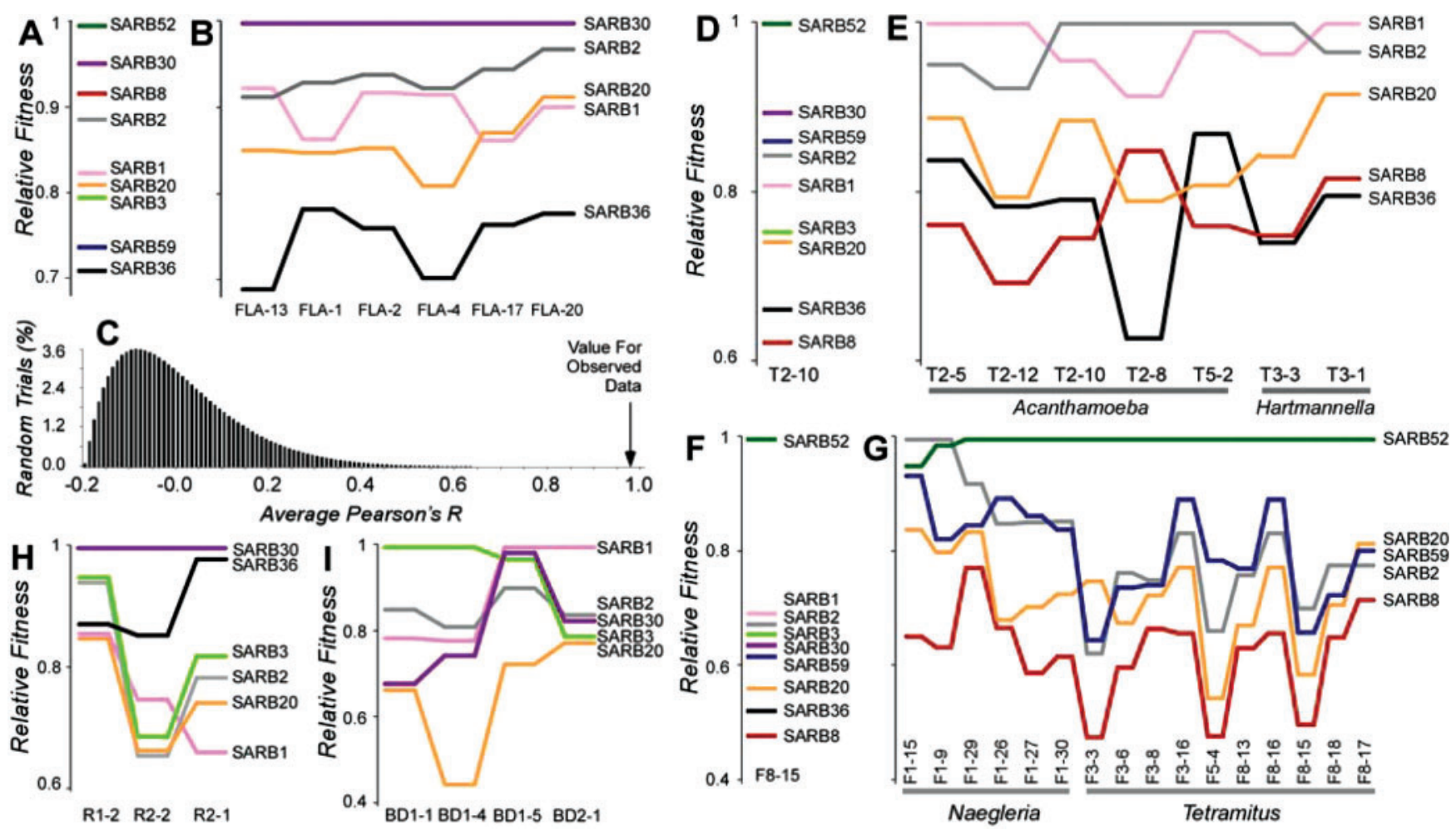

Fig. 1. Feeding preferences of six free-living Acanthamoeba (FLA) isolated from a marine environment (A-C), intestinal amoebae isolated from bullfrog tadpoles $(D, E)$, intestinal amoebae isolated from fish $(F, G)$ and intestinal amoebae isolated from reptiles $(\mathrm{H}, \mathrm{I})$. All preferences were determined using pairwise line tests. (A) Feeding preferences of amoeba FLA-1. The least preferred SARB strain was assigned a fitness of 1.0. (B) Feeding preferences of six FLA amoebae. (C) Distribution of average $R$ values for a randomized set of feeding preferences among sets of nine prey strains and six predators. (D) Feeding preferences of amoeba T2-10. The least preferred SARB strain was assigned a fitness of 1.0. (E) Feeding preferences of seven amoebae isolated from three tadpoles. Amoebae are labelled according to the tadpole from which they were isolated (T2, T3, or T5) followed by a strain number. (F) Feeding preferences of amoeba F8-15. The least preferred SARB strain was assigned a fitness of 1.0. (G) Feeding preferences of 16 amoebae isolated from four goldfish. Amoebae are labelled according to the fish from which they were isolated (F1, F3, F5, or F8) followed by a strain number. (H) Feeding preferences of three amoebae isolated from two turtles. Amoebae are labelled according to the turtle from which they were isolated (R1, R2) followed by a strain number. (I) Feeding preferences of four amoebae isolated from the faeces of two bearded dragons. Amoebae are labelled according to the lizard from which they were isolated (BD1, BD2) followed by a strain number.

Five SARB strains were selected to encompass the range of fitness values observed with FLA-1, and feeding preferences for five other FLA amoebae were determined using these strains (Fig. 1B). The FLA-1 preferences were recapitulated, even for close fitness values, showing that the results of line tests are robust. As a group, all six amoebae show very similar feeding preferences, with SARB30 eaten most slowly, therefore being the most fit of these strains, and SARB36 eaten most quickly and being least fit. The average Pearson correlation coefficient for pairwise comparisons among these profiles showed that the preferences of the six FLA Acanthamoeba strains were quite similar, $R_{\text {Average }}=0.953$. To determine if this average value of $R$ was significantly greater than zero, we devised a randomization test to calculate $P$-values directly (see Methods). For this set of six predators and five prey, the observed similarity of feeding preferences is greater than expected at random
(Fig. 1C; $P<0.0000001$ ). Therefore, we conclude that genetically related amoebae likely residing in the same environment could also share feeding preferences.

\section{Amoebae within tadpoles have similar feeding preferences}

A bacterium may escape a collection of predators in an environment only if those amoebae share feeding preferences. While the results above show that related amoebae recognize the same prey, previous results show that feeding preferences are not shared among unrelated predators from different environments (Wildschutte et al., 2004). For prey bacteria to escape all predators in an environment, even unrelated predators must share feeding preferences. To test this hypothesis, we isolated amoebae from the intestinal tracts of three bullfrog tadpoles, Rana catesbeiana, at 
various stages of development. The smallest tadpole was 2.9 $\mathrm{cm}$ in length and, lacking any limbs, was an herbivorous algavore; the largest tadpole was $3.4 \mathrm{~cm}$ in length and, given both front and rear leg development, this animal was likely transitioning to its adult, carnivorous diet. A total of five strains of Acanthamoeba and two strains of Hartmannella were cultivated and identified based on the sequences of their $18 \mathrm{~S}$ rDNA genes (Table 1). To determine feeding profiles of these predators, we used the strategy outlined above. Acanthamoeba strain T2-10 was first tested using nine SARB strains to determine its feeding preferences (Fig. 1D); to assay additional predators, five SARB strains were chosen to represent the range of fitness values obtained. Strikingly, all seven amoebae isolated from these three tadpoles shared similar feeding preferences (Fig. 1E; $\left.R_{\text {Average }}=0.848, P<0.000004\right)$. These results show that diverse Acanthamoeba from an intestinal environment
- here being up to $20 \%$ different at their rRNA loci collectively prefer one Salmonella serovar over another. More importantly, the feeding preferences of the two Hartmannella strains were similar to those of the Acanthamoeba isolates, even though these genera are distantly related (Fig. 2). These data contrast strongly with the marked differences in feeding preferences for members of these genera isolated from different hosts (Wildschutte et al., 2004), suggesting that feeding profiles may be shared among unrelated amoebae in a particular environment.

\section{Amoebae within fish have similar feeding preferences}

The similarity in feeding preferences among the Acanthamoeba and Hartmannella amoebae isolated from tadpole intestines may reflect either the influence of factors

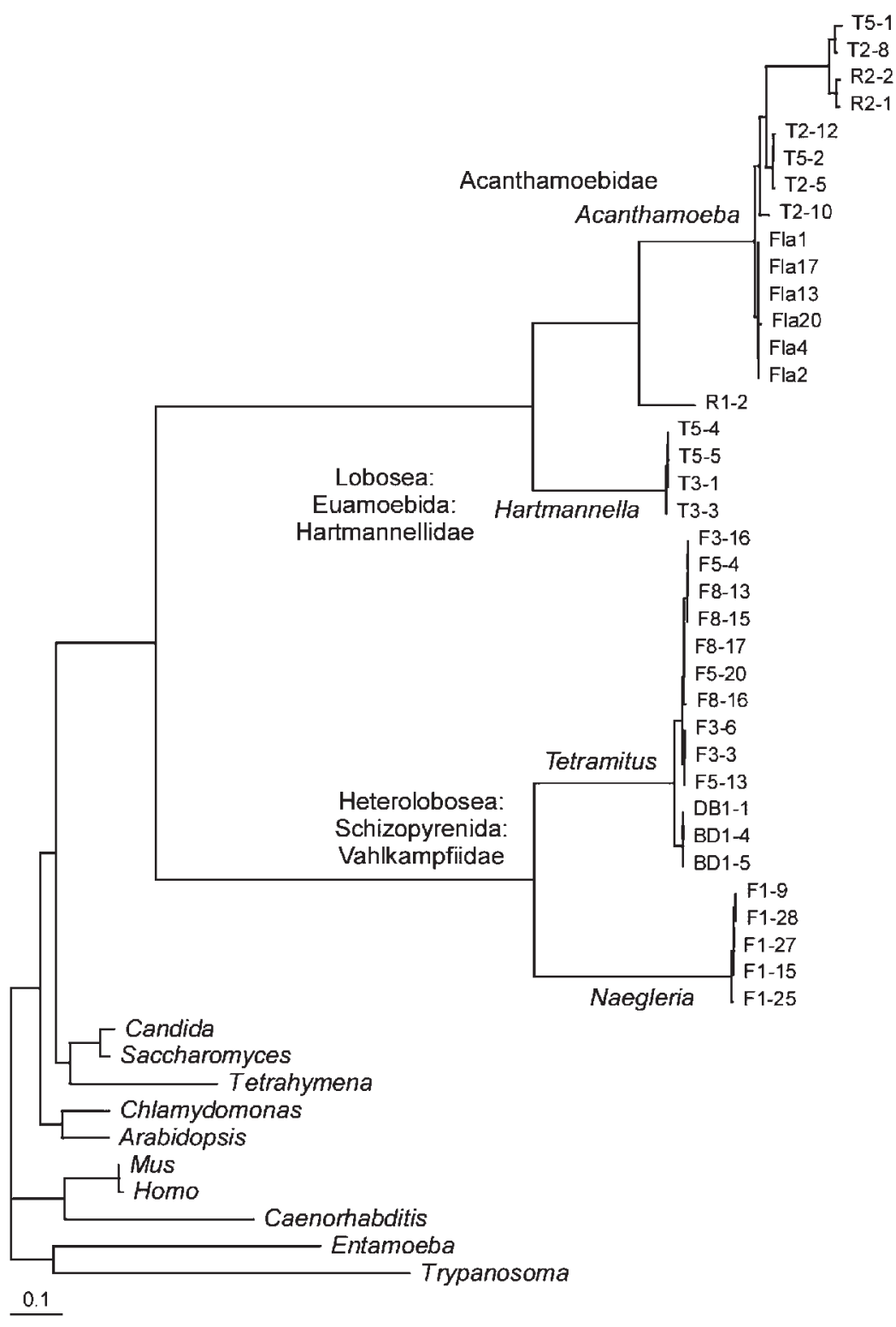

Fig. 2. Phylogeny of $18 \mathrm{~S}$ rDNA loci showing relatedness of amoebae isolated from different hosts; the alignment was not optimized to allow for resolution of deep branches. The dendrogram was constructed using a maximum-likelihood approach implemented by PhyML (Guindon \& Gascuel, 2003). 
within host from which they were isolated or more general, non-host-specific environmental conditions experienced by pond-dwelling creatures, such as the $\mathrm{pH}$ or temperature of the water or the composition of the gut flora. To distinguish between these alternatives, we isolated amoebae from the intestines of goldfish (Carassius auratus auratus) that were reared at two different temperatures and tested their feeding preferences. Fish $\mathrm{F} 1$ was housed at $30{ }^{\circ} \mathrm{C}$; from it we isolated five strains of Naegleria that were $>99 \%$ similar at their $18 \mathrm{~S}$ rDNA loci (Table 1). Three other fish were housed at $23{ }^{\circ} \mathrm{C}$; from them we isolated 11 strains of Tetramitus that were $>98 \%$ similar at their $18 \mathrm{~S}$ rDNA loci (Table 1). Naegleria and Tetramitus are only distantly related, being only $\sim 75 \%$ identical at their $18 \mathrm{~S}$ rDNA loci, despite both typically being classified as members of the family Vahlkampfiidae (Fig. 2). As above, subsets of SARB strains were used to assay the feeding preferences of the 16 amoebae.

The five Naegleria strains from fish $F 1$ all had similar feeding preferences (Fig. 1F, G; $R_{\text {Average }}=0.921$, $P<0.00000001)$. Likewise, the four Tetramitus strains from fish F3 had similar feeding preferences $\left(R_{\text {Average }}=0.955\right.$, $P<0.0007)$ as did the five Tetramitus strains from fish F8 $\left(R_{\text {Average }}=0.913, P<0.000001\right)$. These data reinforce the results obtained with tadpole T2: related amoebae from a single host animal collectively prefer one serovar over another. More importantly, all amoebae isolated from fish - either Naegleria from fish reared at $30{ }^{\circ} \mathrm{C}$ or Tetramitus from fish reared at $23{ }^{\circ} \mathrm{C}$ - shared strongly similar feeding preferences $\left(R_{\text {Average }}=0.897, P<0.0000001\right)$. These data suggest that feeding preferences of commensal, nonpathogenic intestinal amoebae reflect properties of the host, not common environmental conditions.

\section{Fish reared at different temperatures have different microbial flora}

To determine if goldfish reared at different temperatures had dissimilar microbial flora, we isolated Gram-negative bacteria from their intestinal contents. Four strains of Citrobacter were isolated from fish $\mathrm{F} 1$, which was housed at $30{ }^{\circ} \mathrm{C}$ (Table 1); Citrobacter (Enterobacteriaceae) are antigenically diverse bacteria (Knirel et al., 2002) related to Salmonella and E. coli. In contrast, 12 strains of Aeromonas (Aeromonadaceae) were isolated from fish F8, which was housed at $23{ }^{\circ} \mathrm{C}$ (Table 1). A total of 10 strains of Citrobacter and five strains of Aeromonas were isolated from fishes F3 and F5, which were transferred from $30{ }^{\circ} \mathrm{C}$ to $23{ }^{\circ} \mathrm{C}$ and had been housed at $23{ }^{\circ} \mathrm{C}$ for a shorter period of time than had fish F8. We examined the O-antigens of the Citrobacter isolates by gel electrophoresis (Supplementary Fig. S1, available with the online version of this paper). Although the serotype of the O-antigen cannot be determined in this way, one may distinguish between different $\mathrm{O}$-antigens based on the patterns seen in silverstained gels. While strains of Citrobacter exhibit more than 45 different $\mathrm{O}$-antigens, all strains isolated from goldfish carried identical or nearly identical O-antigen carbohydrates, and these differed from the O-antigens of the Aeromonas strains (Supplementary Fig. S1). Together, these data show that amoebae from an intestinal environment have similar feeding preferences, regardless of their relatedness or the identity of prey bacteria, temperature or other environmental factors, strongly suggesting that feeding preferences are a function of the host.

\section{Amoebae from reptiles have similar feeding preferences}

Tadpoles and fish have relatively undifferentiated intestinal tracts. Because enteric bacteria also reside within hosts with more complex intestinal environments, we tested the hypothesis that amoebae from more differentiated intestines also share feeding preferences by isolating amoebae from reptiles. Three strains of Acanthamoeba (R1-2, R2-1 and $\mathrm{R} 2-2 ; 88 \%$ similar at their $18 \mathrm{~S}$ rDNA loci) were isolated from two red-eared sliders, Trachemys scripta (Table 1). Feeding preferences were determined as described above and were again more similar than expected at random (Fig. $1 \mathrm{H} ; R_{\text {Average }}=0.510, P<0.039$ ). Three strains of Tetramitus (BD1-1, BD1-4, BD1-5; >99\% similar at their $18 \mathrm{~S}$ rDNA loci) were isolated from the faeces of a carnivorous, juvenile bearded dragon, Pogona barbata (Table 1), and one strain of Acanthamoeba (BD21) was isolated from an herbivorous adult. Line tests were performed as described previously and the results showed that these amoebae also share feeding preferences (Fig. 1I; $\left.R_{\text {Average }}=0.476, P<0.026\right)$. Thus, amoebae isolated from hosts with differentiated intestines also collectively prefer one Salmonella serovar over another; the larger variation in feeding profiles may reflect the greater diversity of habitats within more differentiated intestines.

\section{DISCUSSION}

\section{Predators form environment-specific threats to particular prey}

Traditionally, microbiologists have viewed antigens - the highly variable proteins and carbohydrates that decorate the surface of bacterial cells - as being intimately associated with the adaptive immune system. These outermost cellular structures are the most accessible to antibodies or white blood cells, so it is not surprising that they are quite variable among bacteria that interact with eukaryotes in this way. The need for systemic pathogens to escape immune recognition continually selects for new, rare antigenic types; here, FDS favours the newly different cells by allowing for prolonged infections or the reinfection of non-naïve hosts (Hosking et al., 1999; Jennings et al., 1995; Reeves, 1995; van Putten, 1993). Yet many non-pathogens are also antigenically diverse, and many pathogens - like Salmonella enterica - have antigenic diversity that is inconsistent with FDS (Wildschutte et al., 2004). For these 
bacteria, we proposed that intestinal protozoa - rather than bacteriophagic white blood cells - are the predators being avoided. Here, a Salmonella serotype could have an advantage in escaping all of the predators in a particular environment; because predators are not uniformly distributed, different prey serotypes would flourish in different environments, a process termed diversifying selection (DS). The finding that amoebae from separate environments have different feeding preferences (Fig. 3) is consistent with this hypothesis. A critical prediction of this model is that predators within particular intestinal environments should share a common set of feeding preferences, thus allowing for some prey to escape predation there better than others.

The data collected here uniformly support this position in five different systems: marine-isolated Acanthamoeba shared similar feeding preferences (Fig. 1B); tadpole-borne Acanthamoeba and Hartmannella also showed significant similarity in prey choice (Fig. 1E); fish-borne Naegleria and Tetramitus showed striking similarity in feeding preferences even though their hosts were reared at different temperatures and harboured different intestinal flora (Fig. 1G; Table 1); and both Acanthamoeba from turtles and Tetramitus and Acanthamoeba from bearded dragons showed similar feeding preferences after isolation from the differentiated colons of their reptilian hosts (Fig. 1H, I). Comparison of Fig. 1 with Fig. 3 highlights the similarity in feeding preferences of co-resident amoebae and the disparity of these preferences of amoebae residing in different environments. It is perhaps not surprising that related amoebae - e.g. those from the same species - would share similar feeding preferences, since the proteins mediating prey recognition would be highly similar. Being closely related, one may expect to find them in the same environment. In addition, the amoebae would have

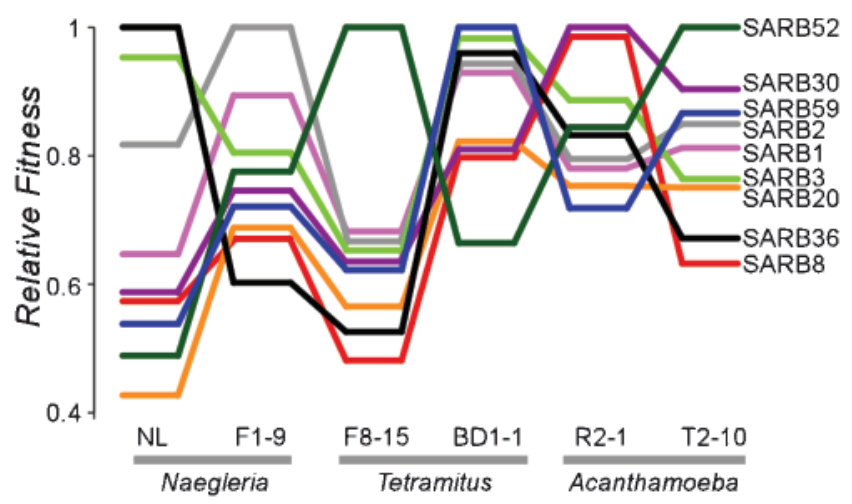

Fig. 3. Feeding preferences of amoebae isolated from different environments; data are gathered from Fig. 1 and from Wildschutte et al. (2004). Each amoeba was tested against nine SARB strains. The feeding preferences of all amoebae were determined by pairwise line tests using nine SARB strains. The least preferred SARB strain was assigned a fitness of 1.0. Amoebae are labelled as previously described; NL is a lab strain of Naegleria. colonized the intestine from the surrounding, geographically constrained environment; as such, they would not represent a random sample of all members of that species. Therefore, one could say that the similar feeding profiles of Acanthamoeba within tadpoles (Fig. 1E) and Tetramitus or Naegleria within fish (Fig. 1G) are not terribly surprising.

But what is unexpected is that amoebae share feeding preferences if and only if they are found in the same environment (Fig. 4). Tetramitus and Naegleria residing within fish share feeding preferences (Fig. 1g), whereas members of these genera residing elsewhere do not (Fig. 3). Unrelated amoebae may be found in a single host due to fluctuating conditions there that may support different flora and fauna at different times. For example, only Hartmannella were isolated from the smallest tadpole, which was likely herbivorous, whereas only Acanthamoeba were isolated from the largest tadpole, whose carnivorous diet would have fostered growth of an entirely different microflora. Similarly, only Naegleria were found in the fish housed at $30{ }^{\circ} \mathrm{C}$, whereas only Tetramitus were isolated from fish housed at lower temperatures; here, different species of enteric bacteria were favoured under the two temperature regimes (Table 1). Yet in both these cases, the unrelated amoebae from each host shared a common set of feeding preferences, despite other major environmental differences between individuals. We infer that there must

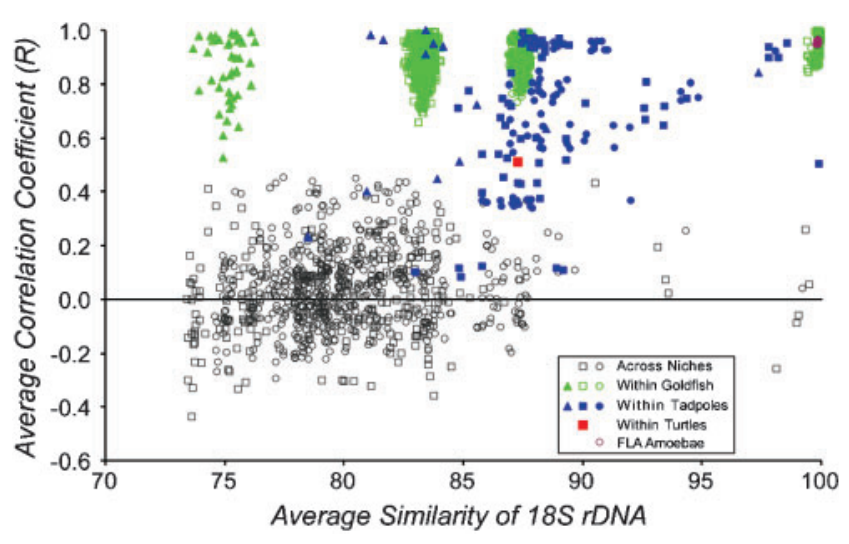

Fig. 4. Similarity of amoeba feeding preferences as a function of genetic relatedness; data are gathered from Fig. 1 and from Wildschutte et al. (2004). Average Pearson's correlation coefficients are reported for groups of two, three and four line tests, plotted against the average similarity at the $18 \mathrm{~S}$ rDNA locus. Comparisons of feeding preferences between amoebae isolated from different environments are shown in grey, among tadpoleisolated amoebae in blue, among goldfish-isolated amoebae in green, among turtle-isolated amoebae in red and among FLA amoebae in violet. Triangles represent comparisons with the feeding preferences of two amoebae; comparisons using three sets of preferences are shown as squares and those using four sets of preferences are shown as circles. Open (transparent) symbols are used when a large density would obfuscate the number of data points. 
be other, host-specific environmental factors within the intestinal lumen that influence the residents. That is, amoebae that do not share these feeding preferences likely do not persist, reflecting selection for sets of unrelated predators with common proclivities in prey choice.

In addition, we might expect amoebae of the same species to have similar feeding preferences, as suggested by the similarity in feeding preferences among closely related Acanthamoeba (Fig. 1B). Yet our data also suggest that related amoebae - up to $99 \%$ at their $18 \mathrm{~S}$ rDNA loci - can have very different feeding preferences when they are found in different environments (grey points in Fig. 4). These related amoebae may be adapted to different environments and again it may be properties of these niches that influence their feeding preferences. Thus, the environment to which an amoeba is adapted may play a pivotal role in shaping its feeding preferences.

\section{Discriminating among environmental carbohydrates}

As the most abundant molecule on the outside of the cell, the bacterial $\mathrm{O}$-antigen is a likely target for amoeba predators; yet amoebae encounter other abundant polysaccharides in their intestinal environments, notably mucins. Mucins are proteins containing variable tandem repeats rich in serine, threonine and proline (Robbe et al., 2003, 2004; Van Klinken et al., 1995); they are heavily substituted with oligosaccharides that are O-linked to serine and threonine residues. Mucins are either secreted or attached to intestinal cells, and their primary functions are thought to be intestinal protection and aiding in gut flora binding (Belley et al., 1999; Deplancke \& Gaskins, 2001; Shirazi et al., 2000; Stanley \& Phillips, 1999). While Oantigens appear on an amoeba's bacterial prey, mucins decorate the intestinal wall; and although bacteria are viable food sources for amoebae, the intestinal wall is not.

We propose that amoebae discriminate among prey because they must differentiate between structures that should and should not be eaten. Recognition of host-specific mucin polysaccharides would allow the amoebae to use these sugars for simple attachment, while avoiding attempts at consuming the intestinal wall. Consistent with this hypothesis, the human-dwelling Entamoeba histolytica has been shown to strongly bind to the abundant mucin sugar $\mathrm{N}$-acetylgalactosamine (Adler et al., 1995; Leroy et al., 1995). This sugar may be a receptor attachment site of E. histolytica, allowing it to reside in its adapted niche and avoid rapid expulsion from the colon. If amoebae differentially bind to mucins, then $\mathrm{O}$ antigens more similar to native intestinal mucins may provide a higher fitness to that bacterium via unintentional mimicry. That is, bacteria whose $\mathrm{O}$-antigens resemble the local mucins may escape predation more readily because they are recognized as housing, not as food. While this model is entirely speculative, it does provide a testable mechanism through which unrelated, co-resident amoebae would share feeding preferences.

\section{Differential distribution of Salmonella may result from predation}

Salmonella serovar-host specificity has historically been viewed as a product of bacterial interaction with host immune systems, whereby a serovar expressing a specific Oantigen could infect a certain host after immune evasion and then cause disease. Previously, we showed that amoebae are a possible selective pressure influencing $\mathrm{O}$-antigen variability (Wildschutte et al., 2004), and here we show that amoebae from certain environments collectively prefer one serovar over another (Fig. 1). Furthermore, amoebae between dissimilar environments have different feeding preference (Figs 3 and 4). As a result, amoeba predation may influence bacterial survival in environments, resulting in the differential distribution of bacteria among hosts: that is, bacteria may be found in an environment because they can survive better against communities of native predators. Under this model, Salmonella serovar-host specificity may have originated after a serovar had established the ability to escape native predators in certain environments. During adaptation to its specific niche, a serovar can acquire genes allowing it to infect that host and cause disease. That is, the specificity for Salmonella in causing disease more readily in particular hosts may be intimately associated with that serovar's ability to avoid the predators within that host; Salmonella must avoid predation before it invades intestinal epithelium. Consistent with this hypothesis, Salmonella and E. coli have been found to be differentially distributed among the intestinal environments of their hosts (Boyd et al., 1993; Gordon \& FitzGibbon, 1999; Gordon et al., 2002; Gordon \& Cowling, 2003; Rabsch et al., 2002). We found similar results here where, for example, the serovars of Salmonella within turtles and bearded dragons were significantly different from those we isolated from birds (Table 1). Because a single $\mathrm{O}$-antigen would not confer high fitness in all environments, $\mathrm{O}$-antigen (and $r f b$ ) variability would be maintained among Salmonella. Thus protozoan predation may be the selective pressure maintaining O-antigen diversity among Salmonella.

\section{ACKNOWLEDGEMENTS}

We thank Sarah Hainer for technical assistance, Dr Todd Katzner for assistance obtaining faecal samples from the Pittsburgh National Aviary and Dr Stanley Reynolds and the Pennsylvania State Health Department for Salmonella serotyping. This work was supported by a grant from the David and Lucile Packard Foundation.

\section{REFERENCES}

Adler, P., Wood, S. J., Lee, Y. C., Lee, R. T., Petri, W. A., Jr \& Schnaar, R. L. (1995). High affinity binding of the Entamoeba histolytica lectin to polyvalent $N$-acetylgalactosaminides. J Biol Chem 270, 5164-5171.

Belley, A., Keller, K., Gottke, M. \& Chadee, K. (1999). Intestinal mucins in colonization and host defense against pathogens. Am J Trop Med Hyg 60, 10-15.

Booton, G. C., Rogerson, A., Bonilla, T. D., Seal, D. V., Kelly, D. J., Beattie, T. K., Tomlinson, A., Lares-Villa, F., Fuerst, P. A. \& Byers, T. J. (2004). Molecular and physiological evaluation of subtropical 
environmental isolates of Acanthamoeba spp., causal agent of Acanthamoeba keratitis. J Eukaryot Microbiol 51, 192-200.

Boyd, E. F., Wang, F.-S., Baltran, P., Plock, S. A., Nelson, K. \& Selander, R. K. (1993). Salmonella reference collection B (SARB): strains of 37 serovars of subspecies I. J Gen Microbiol 139, 1125-1132.

Deplancke, B. \& Gaskins, H. R. (2001). Microbial modulation of innate defense: goblet cells and the intestinal mucus layer. Am J Clin Nutr 73, 1131S-1141S.

Eckburg, P. B., Bik, E. M., Bernstein, C. N., Purdom, E., Dethlefsen, L., Sargent, M., Gill, S. R., Nelson, K. E. \& Relman, D. A. (2005). Diversity of the human intestinal microbial flora. Science 308, 1635-1638.

Gordon, D. M. \& Cowling, A. (2003). The distribution and genetic structure of Escherichia coli in Australian vertebrates: host and geographic effects. Microbiology 149, 3575-3586.

Gordon, D. M. \& FitzGibbon, F. (1999). The distribution of enteric bacteria from Australian mammals: host and geographical effects. Microbiology 145, 2663-2671.

Gordon, D. M., Bauer, S. \& Johnson, J. R. (2002). The genetic structure of Escherichia coli populations in primary and secondary habitats. Microbiology 148, 1513-1522.

Guindon, S. \& Gascuel, O. (2003). A simple, fast, and accurate algorithm to estimate large phylogenies by maximum likelihood. Syst Biol 52, 696-704.

Guttman, D. S. \& Dykhuizen, D. E. (1994). Detecting selective sweeps in naturally occurring Escherichia coli. Genetics 138, 993-1003.

Heinrichs, D. E., Yethon, J. A. \& Whitfield, C. (1998). Molecular basis for structural diversity in the core regions of the lipopolysaccharides of Escherichia coli and Salmonella enterica. Mol Microbiol 30, 221-232.

Hermisson, J. \& Pennings, P. S. (2005). Soft sweeps: molecular population genetics of adaptation from standing genetic variation. Genetics 169, 2335-2352.

Holst, O. \& Brade, H. (1992). Chemical structure of the core region of lipopolysaccharides. In Bacterial Endotoxic Lipopolysaccharides, pp. 134-170. Edited by D. C. Morrison \& J. L. Ryan. Boca Raton, FL: CRC Press.

Hosking, S. L., Craig, J. E. \& High, N. J. (1999). Phase variation of lic1 A, lic2 A and lic3 A in colonization of the nasopharynx, bloodstream and cerebrospinal fluid by Haemophilus influenzae type b. Microbiology 145, 3005-3011.

Jennings, M. P., Hood, D. W., Peak, I. R. A., Virji, M. \& Moxon, E. R. (1995). Molecular analysis of a locus for the biosynthesis and phasevariable expression of the lacto- $N$-neotetraose terminal lipopolysaccharide structure in Neisseria meningitidis. Mol Microbiol 18, 729-740.

Jjemba, P. K. (2001). The interaction of protozoa with their potential prey bacteria in the rhizosphere. J Eukaryot Microbiol 48, 320-324.

Knirel, Y. A., Kocharova, N. A., Bystrova, O. V., Katzenellenbogen, E. \& Gamian, A. (2002). Structures and serology of the O-specific polysaccharides of bacteria of the genus Citrobacter. Arch Immunol Ther Exp (Warsz) 50, 379-391.

Leroy, A., De Bruyne, G., Mareel, M., Nokkaew, C., Bailey, G. \& Nelis, H. (1995). Contact-dependent transfer of the galactose-specific lectin of Entamoeba histolytica to the lateral surface of enterocytes in culture. Infect Immun 63, 4253-4260.

Ley, R. E., Peterson, D. A. \& Gordon, J. I. (2006). Ecological and evolutionary forces shaping microbial diversity in the human intestine. Cell 124, 837-848.

Liu, D., Verma, N. K., Romana, L. K. \& Reeves, P. R. (1991). Relationships among the $r f b$ regions of Salmonella serovars A, B, and D. J Bacteriol 173, 4814-4819.

Matz, C. \& Kjelleberg, S. (2005). Off the hook - how bacteria survive protozoan grazing. Trends Microbiol 13, 302-307.
Milkman, R., Jaeger, E. \& McBride, R. D. (2003). Molecular evolution of the Escherichia coli chromosome. VI. Two regions of high effective recombination. Genetics 163, 475-483.

Palmer, C., Bik, E. M., Eisen, M. B., Eckburg, P. B., Sana, T. R., Wolber, P. K., Relman, D. A. \& Brown, P. O. (2006). Rapid quantitative profiling of complex microbial populations. Nucleic Acids Res 34, e5.

Peduzzi, P. \& Schiemer, F. (2004). Bacteria and viruses in the water column of tropical freshwater reservoirs. Environ Microbiol 6, 707-715.

Pernthaler, J. (2005). Predation on prokaryotes in the water column and its ecological implications. Nat Rev Microbiol 3, 537-546.

Popoff, M. Y. (2001). Antigenic Formulas of the Salmonella Serovars, 8th edn. Paris: Institut Pasteur.

Rabsch, W., Andrews, H. L., Kingsley, R. A., Prager, R., Tschape, H., Adams, L. G. \& Baumler, A. J. (2002). Salmonella enterica serotype Typhimurium and its host-adapted variants. Infect Immun 70, 2249-2255.

Reeves, P. (1995). Role of O-antigen variation in the immune response. Trends Microbiol 3, 381-386.

Robbe, C., Capon, C., Flahaut, C. \& Michalski, J. C. (2003). Microscale analysis of mucin-type O-glycans by a coordinated fluorophoreassisted carbohydrate electrophoresis and mass spectrometry approach. Electrophoresis 24, 611-621.

Robbe, C., Capon, C., Coddeville, B. \& Michalski, J. C. (2004). Structural diversity and specific distribution of O-glycans in normal human mucins along the intestinal tract. Biochem J 384, 307-316.

Ronn, R., McCaig, A. E., Griffiths, B. S. \& Prosser, J. I. (2002). Impact of protozoan grazing on bacterial community structure in soil microcosms. Appl Environ Microbiol 68, 6094-6105.

Shirazi, T., Longman, R. J., Corfield, A. P. \& Probert, C. S. (2000). Mucins and inflammatory bowel disease. Postgrad Med J 76, 473-478.

Stanley, C. M. \& Phillips, T. E. (1999). Selective secretion and replenishment of discrete mucin glycoforms from intestinal goblet cells. Am J Physiol 277, G191-G200.

Stenutz, R., Weintraub, A. \& Widmalm, G. (2006). The structures of Escherichia coli O-polysaccharide antigens. FEMS Microbiol Rev 30, 382-403.

Van Klinken, B. J., Dekker, J., Buller, H. A. \& Einerhand, A. W. (1995). Mucin gene structure and expression: protection vs. adhesion. Am J Physiol 269, G613-G627.

van Putten, J. P. (1993). Phase variation of lipopolysaccharide directs interconversion of invasive and immuno-resistant phenotypes of Neisseria gonorrhoeae. EMBO J 12, 4043-4051.

Wang, L., Romana, L. K. \& Reeves, P. R. (1992). Molecular analysis of a Salmonella enterica group E1 rfb gene cluster: O-antigen and the genetic basis of the major polymorphism. Genetics 130, 429-443.

Whitfield, C. \& Roberts, I. S. (1999). Structure, assembly and regulation of expression of capsules in Escherichia coli. Mol Microbiol 31, 1307-1319.

Whitman, W. B., Coleman, D. C. \& Wiebe, W. J. (1998). Prokaryotes: the unseen majority. Proc Natl Acad Sci U S A 95, 6578-6583.

Wildschutte, H., Wolfe, D. M., Tamewitz, A. \& Lawrence, J. G. (2004). Protozoan predation, diversifying selection, and the evolution of antigenic diversity in Salmonella. Proc Natl Acad Sci U S A 101, 10644-10649.

Xiang, S. H., Haase, A. M. \& Reeves, P. R. (1993). Variation of the $r f b$ gene clusters in Salmonella enterica. J Bacteriol 175, 4877-4884.

Edited by: J. Tachezy 